\title{
Predictive Model for Adverse Events and Immune Response Based on the Production of Antibodies After the Second-Dose of the BNT162b2 mRNA Vaccine
}

\author{
Shinichi Okada,* Katsuyuki Tomita,, Genki Inui, $\uparrow$ Tomoyuki Ikeuchi, $\uparrow$ Hirokazu Touge, $\uparrow$ Junichi Hasegawa $\$$ and \\ Akira Yamasaki§ \\ *Department of Pediatrics, National Hospital Organization Yonago Medical Center, Yonago 683-0006, Japan, †Department of \\ Respiratory Medicine, National Hospital Organization Yonago Medical Center, Yonago 683-0006, Japan, †Department of Internal \\ Medicine, National Hospital Organization Yonago Medical Center, Yonago 683-0006, Japan, and \$Division of Respiratory Medicine \\ and Rheumatology, Department of Multidisciplinary Internal Medicine, School of Medicine, Faculty of Medicine, Tottori University, \\ Yonago 683-8503, Japan
}

\section{ABSTRACT}

Background The BNT162b mRNA vaccine for coronavirus disease 2019, which is caused by severe acute respiratory syndrome coronavirus 2 (SARS-CoV-2), mimics the immune response to natural infection. Few studies have predicted the adverse effects (AEs) after the second-dose vaccination. We present a predictive model for AEs and immune response after the second-dose of the BNT162b mRNA vaccine.

Methods To predict AEs, 282 healthcare workers (HCWs) were enrolled in this prospective observational study. The classification and regression tree (CART) model was established, and its predictive efficacy was assessed. To predict immune response, $282 \mathrm{HCWs}$ were included in the analysis. Moreover, the factors affected by anti-SARS-CoV-2 spike protein RBD antibody (sIgG) were evaluated using serum samples collected 2 months after the second-dose vaccination. The s-IgG level was assessed using Lumipulse G1200. Multiple regression analyses were conducted to evaluate variables associated with anti-s-IgG titer levels.

Results The most common AEs after the seconddose vaccination were pain $(87.6 \%)$, redness $(17.0 \%)$ at the injection site, fatigue $(68.8 \%)$, headache $(53.5 \%)$, and fever (37.5\%). Based on the CART model, headache after the first-dose vaccination and age $<30$ years were identified as the first and second discriminators for predicting the headache after the second-dose vaccination, respectively. In the multiple linear regression model, anti-s-IgG titer levels were associated with age, female sex, and AEs including headache and induration at the injection site after the second-dose vaccination.

Conclusion Headache after the first-dose vaccination can be a predictor of headache after the seconddose vaccination, and AEs are indicators of immune response.

Key words adverse effect; antibody; BNT162b2 vaccine; classification and regression tree
Coronavirus disease 2019 (COVID-19) is caused by severe acute respiratory syndrome coronavirus 2 (SARS-CoV-2). A COVID-19 pandemic has emerged globally. ${ }^{1}$ It caused unprecedented medical, social, and economic damages worldwide, with more than 186 million people infected and over 4 million deaths recorded since July 1, 2021. ${ }^{2}$ Developing vaccines against COVID-19 is critical for eradicating this pandemic. Several vaccines have been rolled out in various countries. ${ }^{2}$ To lessen the burden on healthcare systems, massive vaccination is a top global priority.

Among these vaccines is the lipid nanoparticleformulated, BNT162b2 nucleoside-modified RNA vaccine (BNT162b2; Pfizer-BioNTech NY, the USA, and Mainz, German). It encodes a membrane-anchored SARS-CoV-2 full-length spike protein stabilized in the perfusion conformation. Polack and colleagues have revealed that the efficacy rate of the BNT162b2 mRNA vaccine after two doses was $94.8 \% .^{3}$ The main adverse effects (AEs) of this vaccine are generalized weakness or fatigue $(58.9 \%)$, headache $(44.8 \%)$, chills $(36.0 \%)$, fever $(22.0 \%)$, sweating $(9.2 \%)$, dizziness $(8.3 \%)$, and flushing (7.1\%). ${ }^{4}$ In addition, the localized symptoms are sore arm $(88.0 \%)$, localized swelling at the injection site (5.5\%), and itching (5.4\%). ${ }^{4}$ These AEs are more common after the second-dose vaccination. ${ }^{5}$ Nevertheless, systemic symptoms such as fever and fatigue can decrease work productivity and increase the need for medical attention. However, data about the prediction of AEs are limited. Hence, the current study aimed to analyze the predictive factors for AEs after the second-dose

Shinichi Okada and Katsuyuki Tomita contributed equally to this work.

Corresponding author: Katsuyuki Tomita, MD, PhD

ktomita0223@gmail.com

Received 2021 October 25

Accepted 2022 January 5

Online published 2022 February 22

Abbreviations: AEs; adverse effects, AUC; area under the receiver operating curve, CART; classification and regression tree, COVID-19; Coronavirus disease-19, HCWs; healthcare workers, SARS-CoV-2; severe acute respiratory syndrome coronavirus 2 
of the BNT162b2 mRNA vaccine using a self-reported questionnaire.

After two doses, the BNT162b2 mRNA vaccine induced a strong antibody response to the antiSARS-CoV-2 spike protein as anti-SARS-CoV-2 spike IgG (anti-s-IgG). However, the high titer levels of antis-IgG decreased 1.5 months after the second-dose vaccination. ${ }^{6}$ The BNT162b2 mRNA vaccine may closely mimic the immune response to natural infection. ${ }^{7} \mathrm{We}$ hypothesized that several AEs are correlated with immune response after vaccination. Therefore, this study also investigated the association between anti-s-IgG titer levels and AEs.

\section{MATERIALS AND METHODS \\ Study design}

This prospective observational cohort study assessed all adverse events after the first-dose of the BNT162b2 vaccine from February 19, 2021, to April 9, 2021. Of 547 healthcare workers (HCWs) at National Hospital Organization Yonago Medical Center, 497 received the first dose of the BNT162b2 vaccine. Of them, 494 had the second-dose at an interval of 3 weeks. Then, a selfreported questionnaire of AEs was administered to 282 participants. The inclusion criteria were as follows: (1) age 20 years or older and (2) consent to participate in the study. This study was approved by the institutional review board of the institution (no. 0305-06). A written informed consent was obtained from all participants after they received an explanation about the purpose and procedures of the study.

\section{Assessment of AEs}

All participants who were vaccinated with the first dose were informed about the risk of AEs after the vaccination, and they received a self-reported paper-based questionnaire concerning AEs. The AEs after the first and second-dose of the vaccine were monitored using the questionnaire. It included three items for generalized symptoms (weakness including fatigue, headache, and fever) and four items for symptoms localized at the injection site (pain, redness, swelling, and induration). Body temperature and the radii of redness, swelling and induration were measured twice a day (morning and evening). Along with this, the AEs were recorded daily as well as symptoms as AEs. The questionnaire included questions on the presence or severity of AEs as well as on the intensity of the reaction; it graded the severity into mild (no interfere with activity), moderate (some interfere with activity), severe (preventing daily activity), and, critical (emergency room visit or hospitalization). One week and one month after each vaccination, all reports were collected for analysis, and the following data were obtained for each report: date reported, age and sex of the participants, comorbidities, AEs, time interval between the vaccination and reaction.

\section{Measurement of anti-SARS-CoV-2 Spike IgG titer levels}

The participants signed an informed consent form for sampling and the usage of clinical data. Blood samples were collected 2 months after the second- dose vaccination. Chemiluminescent enzyme immunoassay (CLEIA) was performed using Lumipulse G1200 (Fujirebio, Tomita, Japan), which can assay 120 samples per hour for the qualitative detection of IgG against SARS-CoV-2 spike protein (anti-s-IgG). The cutoff value for this assay was $1.0 \mathrm{AU} / \mathrm{mL}$, with $<1.0 \mathrm{AU} / \mathrm{mL}$ indicating a negative result. The maximum value was $60 \mathrm{AU} / \mathrm{mL}$. If values exceeded the detection limit, the samples were diluted with phosphate-buffered solution.

\section{Statistical analysis}

While making a decision, classification and regression tree (CART) model usually mimics human thinking ability using if-else structures. The CART is non-parametric; therefore, it does not depend on the information obtained from a particular type of distribution and is not impacted by outliers in the input variables. The CART model was built to predict AEs after the second-dose of vaccination, using various parameters of age, sex, and AEs after first dose of vaccination as the variable factors. We developed the CART model using R package "rpart" and "partykit". ${ }^{-10}$ The tree model could predict the outcome using a set of if-else questions. The regression tree analysis was performed to predict the outcome as a real number. CART builds a tree via repeated partitioning. Hence, the data set was successfully split into increasingly homogenous subgroups. The outcomes in each final subspace were as homogeneous as possible. At each stage (node), the CART algorithm selected the explanatory variable and splitting value that provided the more significant variables to distinguish each final subspace. The full CART algorithm added nodes until they were homogenous or contained few observations (cutoff of $\geq 5$ ). Then, the CART model was used to split data: $80 \%$ to the test samples and $20 \%$ to the validated samples. To assess the performance of the models, the area under the receive operating characteristic curve (AUC) was calculated using R ("pROC"). ${ }^{11}$

A multiple linear regression analysis was performed to develop a model for elucidating the relationship between anti-s-IgG titer levels and variable factors. 

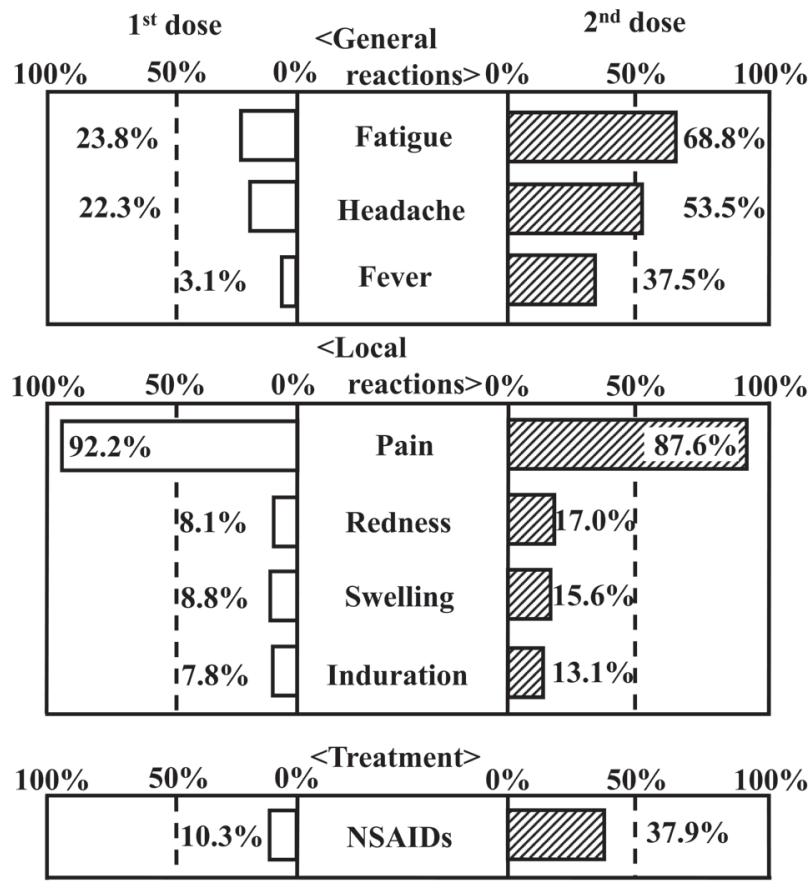

Fig. 1. Prevalence of adverse effects after the first (white bar) and second (hatched bar) dose of the BNT162b2 vaccine.

Normal QQ plot tool was used to assess their effect on the normality of the distribution of the data.

All data analyses to make graphs were performed using the R package "ggplot2". Relative risk was calculated by dividing the risk of AEs in a specific population group by the risk of AEs from all other groups.

\section{RESULTS}

\section{AEs after the BNT162b2 mRNA vaccination}

In total, 282 individuals were included in the analysis of AEs after the first-dose vaccination. The mean age of the participants was 38 (range: $20-77$ ) years, and $74 \%$ of the study population were women. Approximately $94 \%$ of participants reported some types of AEs after the first-dose vaccination. The most common AEs were pain $(92.2 \%)$, swelling $(8.8 \%)$, redness $(8.1 \%)$ or induration $(7.8 \%)$ at the injection site, fatigue $(23.8 \%)$, headache (22.3\%), and fever (3.1\%) (Fig. 1). Next, 282 participants were assessed for AEs after the seconddose vaccination. The common AEs were pain (87.6\%), redness $(17.0 \%)$, swelling $(15.6 \%)$ or induration $(13.1 \%)$ at the injection site, fatigue $(68.8 \%)$, headache $(53.5 \%)$, and fever $(37.5 \%)$. Moreover, the prevalence of AEs after the second-dose vaccination was higher than that after the first-dose vaccination (Fig. 1). Most reported events were mild to moderate, and they occurred within $48 \mathrm{~h}$ post-vaccination. However, $24(8.5 \%)$ participants reported one or several symptoms that significantly
(A)

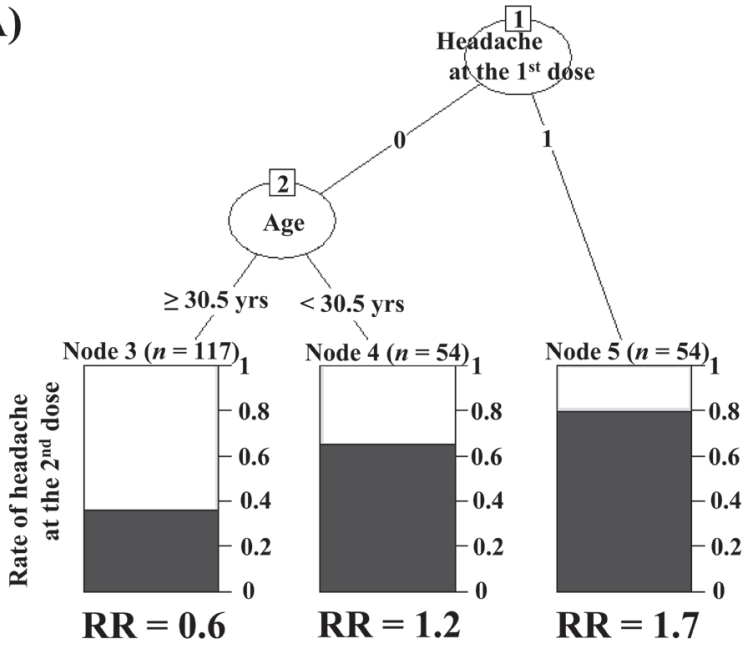

(B)

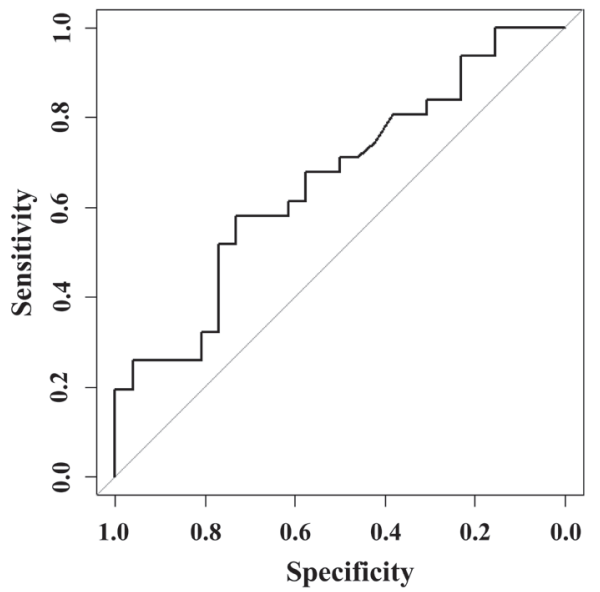

Fig. 2. Decision tree generated by Classification and Regression Tree (CART) model for predicting headache after the second-dose of the BNT162b2 vaccine (A), and validation of the prediction mode using ROC (B). The relative risks are given at each leaf. yrs, years.

disturbed activities of daily living. Moreover, the symptoms after the second-dose vaccination lasted for several days and/or caused absence from work.

\section{Factors influencing AEs after the second-dose vaccination}

We used the predictive model for AEs after the seconddose of the vaccination where headache was the only $\mathrm{AE}$ that could be predicted and the other AEs were not identified by the CART model. Figure 2A depicts the CART model, which was presented as a tree diagram. To predict headache after the second-dose vaccination, the first split was based on whether vaccinated individuals presented with headache after the first-dose vaccination (node $1, \mathrm{RR}=1.7$ ). The node representing headache 
Table 1 . Parameter estimates of anti-s-IgG titer levels 2 months after the second-dose of the BNT162b2 vaccine according to demographic variables via a linear univariate analysis

\begin{tabular}{|c|c|c|c|c|c|c|}
\hline \multirow[b]{2}{*}{ Parameter } & \multirow[b]{2}{*}{ Estimates } & \multirow[b]{2}{*}{ Std. Error } & \multirow[b]{2}{*}{$t$} & \multirow[b]{2}{*}{ Sig. } & \multicolumn{2}{|c|}{$95 \% \mathrm{CI}$} \\
\hline & & & & & $\begin{array}{l}\text { Lower } \\
\text { Bound }\end{array}$ & $\begin{array}{l}\text { Upper } \\
\text { Bound }\end{array}$ \\
\hline Male sex $=1$ & -30.1 & 7.7 & -3.9 & $<0.01$ & -45.3 & -14.9 \\
\hline Age & -1.2 & 0.3 & -4.4 & $<0.01$ & -1.8 & -0.7 \\
\hline Fatigue after the second-dose: Yes = 1 & 23.4 & 7.4 & 3.2 & $<0.01$ & 8.8 & 38.0 \\
\hline Headache after the second-dose: Yes $=1$ & 29.8 & 6.7 & 4.4 & $<0.01$ & 16.6 & 43.0 \\
\hline Fever after the second-dose: Yes $=1$ & 22.4 & 7.0 & 3.2 & $<0.01$ & 8.6 & 36.2 \\
\hline Pain after the second-dose: Yes $=1$ & -2.5 & 11.1 & -0.2 & 0.83 & -24.3 & 19.4 \\
\hline Redness after the second-dose: Yes $=1$ & 15.7 & 9.4 & 1.7 & 0.10 & -2.9 & 34.3 \\
\hline Swelling after the second-dose: Yes $=1$ & 19.9 & 9.8 & 2.0 & 0.04 & 0.7 & 39.2 \\
\hline Induration at the injection site after the second-dose: Yes $=1$ & 31.2 & 10.0 & 3.1 & $<0.01$ & 11.4 & 51.0 \\
\hline Use of NSAIDs after the second-dose: Yes $=1$ & 10.2 & 7.1 & 1.4 & 0.15 & -3.8 & 24.1 \\
\hline
\end{tabular}

CI, confidence interval; NSAIDs, nonsteroidal anti-inflammatory drugs; Sig., significance; Std., standard.

after the first-dose vaccination was further split according to age $<30$ years) (node 2, $R R=1.2$ ). Therefore, the analysis resulted in three terminal nodes. The validated CART model created by the combination of AE after the first-dose vaccination and age had a satisfactory performance, with an AUC of $66 \%$ (Fig. 2B). There was no relationship between the occurrence of AEs after the second-dose vaccination and the severity of any $\mathrm{AE}$ after the first-dose of vaccination.

\section{Anti-SARS-CoV-2 spike IgG titer levels}

In total, 381 participants received their first and second doses of the vaccine, and their blood samples were collected post-vaccination. Among them, 278 completed the questionnaire about post-vaccination AEs. The eligible participants were aged $39.5 \pm 11.6$ years, and about $76.0 \%$ were women. The mean anti-s-IgG titer level was $85.3 \pm 52.2 \mathrm{AU} / \mathrm{mL}$.

\section{Factors influencing the immune response}

There was a significant negative correlation between age and s-IgG response 2 months after the second-dose vaccination. First, the linear univariate regression model of anti-s-IgG titer levels was assessed 2 months after the second-dose vaccination (Table 1). Results showed that the parameters for predicting s-IgG response were sex, age, fatigue, headache, fever, swelling, and induration at the injection site after the second-dose vaccination.

Based on the result of the regression analysis of s-IgG response according to age and sex, which are predictor variables, the regression equations of female and male participants (Fig. 3A) were as follows:
For female participants, the log-transformed anti-sIgG titer level $=2.091-0.005^{*}$ (age).

For male participants, the log-transformed anti-sIgG titer level $=1.936-0.005^{*}$ (age).

Based on the results of the regression analysis of ant-s-IgG titer levels according to age and headache, which are predictor variables, after the second-dose vaccination, the regression equations of participants with and without headache after the second-dose vaccination (Fig. 3B) were as follows:

In individuals with headache $(+)$, the log-transformed anti-s-IgG titer level $=2.156-0.006^{*}$ (age).

In individuals without headache (-), the logtransformed anti-s-IgG titer level $=1.900-0.004^{*}$ (age).

Based on the result of the regression analysis of anti-s-IgG titer levels according to age and induration at the injection site, which are predictor variables, after the second-dose vaccination, the regression equations of participants with and without induration at the injection site after the second-dose vaccination (Fig. 3C) were as follows:

In patients with induration at the injection site $(+)$, the log-transformed anti-s-IgG titer level $=2.063$ $-0.006^{*}$ (age).

In patients without induration at the injection site $(-)$, the log-transformed anti-s-IgG titer level $=2.156$ $-0.006^{*}$ (age).

Second, according to the lowest Akaike's Information Criterion score, some parameters of age, sex, and induration and headache after the second-dose of vaccination were included in a stepwise multiple linear regression analysis. The normality of the distribution 


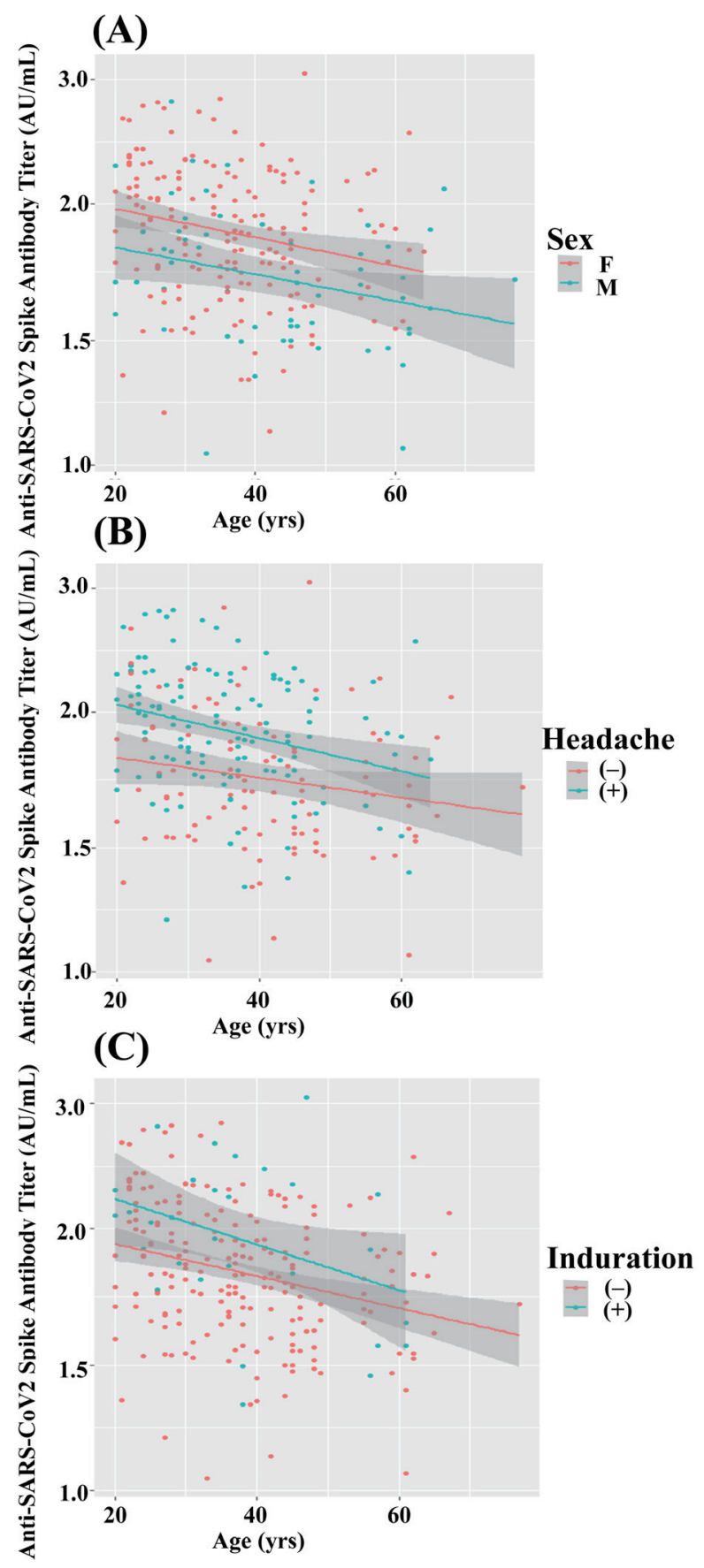

Fig. 3. Association between anti-SARS-CoV2 spike antibody (s$\mathrm{IgG}$ ) titer levels 2 months after the second-dose of the BNT162b2 vaccine and age. Scatter plot and regression equations with $95 \%$ confidence intervals according to sex (A), headache (B), and induration at the injection site $(\mathbf{C})$ after the second-dose of the BNT162b2 vaccine. The vertical axis is on a logarithmic scale. F, female; $\mathrm{M}$, male; yrs, years.

of the data was confirmed using the normal QQ plot tool.

The final participant-predicted equation of anti-sIgG was as follows:
The log-transformed anti-s-IgG titer level $=4.49$ $-0.01 *$ (age) $-0.24 *$ (male) $+0.23 *$ (induration at the injection site after the second-dose vaccination) + $0.30 *$ (headache after the second-dose vaccination).

\section{DISCUSSION}

This study showed that headache after the first-dose of the BNT162b2 vaccine and age $<30$ years could be a predictor of headache after the second-dose vaccination based on the CART model. Moreover, anti-s-IgG titer levels might be associated with younger age, female, and incidences of AEs such as headache and induration at the injection site after the second-dose vaccination.

In our study, headache was the only $\mathrm{AE}$ after the second-dose of vaccine that could be predicted, and the other AEs were not identified by the CART model. The most common side effects of the BNT162b2 vaccine were headache, fatigue, muscle pain, and nausea, with approximately $40 \%$ of individuals presenting with headache. ${ }^{4}$ Patients infected with COVID-19 who developed headache, which is an early symptom of infection, had a milder illness and less risk for mortality. ${ }^{12} \mathrm{We}$ demonstrated that the factors correlated with headache after the second-dose vaccination were a history of headache after the first-dose vaccination and age $<30$ years. The reason is because headache after the first-dose vaccination was supposed to provide information about the ability of the vaccine to induce similar reactions against COVID-19 infection.

Our result was consistent with that of a previous report showing a negative correlation between antibody responses and age among vaccinated individuals. ${ }^{6}$ Age is an important factor influencing vaccine responses. Elderly individuals develop lower antibody levels. Hence, they are poor response to influenza, ${ }^{13}$ hepatitis $\mathrm{A}$ and $\mathrm{B},{ }^{14}$ and pneumococcal vaccines. ${ }^{15}$ In agreement to the recent findings, ${ }^{16}$ we showed that the postvaccination anti-s-IgG titer were higher in females than those in males. It was reported that female patients with COVID-19 infection had more robust $\mathrm{T}$ cell activation than male patients during COVID-19 infection. ${ }^{17}$ As BNT162b2 vaccine elicited a strong TH1-biased CD4+ $\mathrm{T}$ cell response and IFN $\gamma+\mathrm{CD} 8+\mathrm{T}$ cell response in nonhuman primates, ${ }^{18}$ the sex-dependent antibody response after the vaccination might attribute to this difference of $\mathrm{T}$ cell responses between males and females.

It was reported that anti-s-IgG might contribute to the prevention of COVID-19 infection, ${ }^{19}$ and the lower titers of anti-s-IgG could predict COVID-19 infection after vaccination, also known as the break-through infection. Further, anti-s-IgG persistence and titers are based on the severity of the COVID-19 infection. ${ }^{20}$ In 
this study, the occurrence of AEs after the second-dose of vaccination is a useful indicator of developing a rapid immune response to the vaccine. Some AEs including headache and induration at the injection site seen after the second-dose vaccination were independent factors for predicting anti-s-IgG titer after the second-dose of vaccination.

A previous study found that NSAIDs including ibuprofen may reduce the production of antibodies and affect the immune response to the virus itself. ${ }^{21}$ However, our data showed that NSAIDs did not affect the production of anti-s-IgG.

The current study had some limitations. First, it was conducted at a single center, and a small number of participants was included. Second, cellular immunity was not evaluated, and the predictive efficacy was evaluated only according to antibody levels. Third, the anti-s-IgG titer level was only assessed once. As the antibody response to the spike protein began 1.5 months after the second-dose vaccination, ${ }^{22}$ our measuring time-point was almost peak of anti-s-IgG titer.

Given that vaccination indicates mimicking of the actual virus, it also triggers an immune response that may resemble responses triggered by COVID-19 infection, causing similar ailments in the body. Headache is a frequent symptom following COVID-19 immunization with a typical onset after the vaccination. Therefore, headache after the first-dose vaccination can predict AEs after the second-dose vaccination. Further, headache after the second-dose vaccination can predict immune response two months after the second-dose of vaccination. These results were useful in explaining the vaccinated individuals that $\mathrm{AEs}$ indicate the activation of immune responses with increased anti-s-IgG.

Acknowledgments: We are grateful to Miki Ogi, who is an infectious control nurse at our institution, for providing assistance in participant enrollment.

The authors declare no conflicts of interest.

\section{REFERNCES}

1 Novelli G, Biancolella M, Mehrian-Shai R, Erickson C, Pollitt $\mathrm{KG}$, Vasilion V, et al. COVID-19 update: the first 6 months of the pandemic. Hum Genomics. 2020;14:48. DOI: 10.1186/ s40246-020-00298-w

2 Coronavirus disease (COVID-19) pandemic [Internet]. Worldometer [cited 2021 Jul 9]. Available from: https://www. worldometers.info/coronavirus/?\#countries

3 Polack FP, Thomas SJ, Kitchin N, Absalon J, Gurtman A, Lockhart S, et al.; C4591001 Clinical Trial Group. Safety and Efficacy of the BNT162b2 mRNA Covid-19 Vaccine. N Engl J Med. 2020;383:2603-15. DOI: 10.1056/NEJMoa2034577, PMID: 33301246
4 Kadali RAK, Janagama R, Peruru S, Malayala SV. Side effects of BNT162b2 mRNA COVID-19 vaccine: A randomized, cross-sectional study with detailed self-reported symptoms from healthcare workers. Int J Infect Dis. 2021;106:37681. DOI: 10.1016/j.ijid.2021.04.047, PMID: 33866000

5 Pfizer-BioNTech COVID-19 vaccine overview and safety [Internet]. Atlanta: Centers for Disease Control and Prevention [cited $2021 \mathrm{Jul}$ 7]. Available from: https://www.cdc.gov/ coronavirus/2019-ncov/vaccines/different-vaccines/PfizerBioNTech.html

6 Naaber P, Jürjenson V, Adamson A, Sepp E, Tserel L, Kisand $\mathrm{K}$, et al. Antibody response after COVID-19 mRNA vaccination in relation to age, sex, and side effects. medRxiv. 2021. DOI: 10.1101/2021.04.19.21255714.

7 Wang Z, Schmidt F, Weisblum Y, Muecksch F, Barnes $\mathrm{CO}$, Finkin $\mathrm{S}$, et al. mRNA vaccine-elicited antibodies to SARS-CoV-2 and circulating variants. Nature. 2021;592:61622. DOI: 10.1038/s41586-021-03324-6, PMID: 33567448

8 Breiman L, Friedman R, Olshen A, Stone CJ. (1984) Classification and regression trees. Monterey: Wadsworth \& Brooks/ Cole. p. 358.

9 Therneau T, Atkinson B, Repley B. Package 'rpart' [Internet]. 2019 Apr 12 [cited 2021 Jul 18]. Available from: https://cran.rproject.org/web/packages/rpart/rpart.pdf

10 Hothorn T, Seibold H, Zeileis A. Package 'partykit' [Internet]. 2021 Mar 3 [cited 2021 Jul 18]. Available from: https://cran.rproject.org/web//packages/partykit/partykit.pdf

11 Robin X, Turck N, Hainard A, Tiberti N, Lisacek F, Sanchez J-C, et al. Package 'pROC' [Internet]. 2021 Jan 13 [cited 2021 Jul 18]. Available from: https://cran.r-project.org/web/packages/pROC/pROC.pdf

12 Gonzalez-Martinez A, Fanjul V, Ramos C, Serrano Ballesteros J, Bustamante M, Villa Martí A, et al. Headache during SARS-CoV-2 infection as an early symptom associated with a more benign course of disease: a case-control study. Eur J Neurol. 2021;28:3426-36. DOI: 10.1111/ene.14718, PMID: 33417287

13 Bernstein E, Gardner EM, Abrutyn E, Gross P, Murasko DM. Cytokine production after influenza vaccination in a healthy elderly population. Vaccine. 1998;16:1722-31. DOI: 10.1016/ S0264-410X(98)00140-6, PMID: 9778748

14 Van der Wielen M, Van Damme P, Chlibek R, Smetana J, von Sonnenburg F. Hepatitis A/B vaccination of adults over 40 years old: comparison of three vaccine regimens and effect of influencing factors. Vaccine. 2006;24:5509-15. DOI: 10.1016/j.vaccine.2006.04.016, PMID: 16725234

15 Rubins JB, Puri AKG, Loch J, Charboneau D, MacDonald R, Opstad N, et al. Magnitude, duration, quality, and function of pneumococcal vaccine responses in elderly adults. J Infect Dis. 1998;178:431-40. DOI: 10.1086/515644, PMID: 9697723

16 Terpos E, Trougakos IP, Apostolakou F, Charitaki I, Sklirou AD, Mavrianou N, et al. Age-dependent and genderdependent antibody responses against SARS-CoV -2 in health workers and octogenarians after vaccination with the BNT162B2 mRNA vaccine. Am J Hematol. 2021;96:E257-9. DOI: 10.1002/ajh.26185, PMID: 33837984

17 Takahashi T, Ellingson MK, Wong P, Israelow B, Lucas C, Klein J, et al.; Yale IMPACT Research Team. Sex differences in immune responses that underlie COVID-19 disease outcomes. Nature. 2020;588:315-20. DOI: 10.1038/s41586-0202700-3, PMID: 32846427 
18 Vogel AB, Kanevsky I, Che Y, Swanson KA, Muik A, Vormehr M, et al. BNT162b vaccines protect rhesus macaques from SARS-CoV-2. Nature. 2021;592:283-9. DOI: 10.1038/s41586-021-03275-y, PMID: 33524990

19 Kato H, Miyakawa K, Ohtake N, Yamaoka Y, Yajima S, Yamazaki E, et al. Vaccine-induced humoral and cellular immunity against SARS-CoV-2 at 6 months post BNT16b2 vaccination. medRxiv. 2021. DOI: 10.1101/2021.10.30.21265693.

20 Yan X, Chen G, Jin Z, Zhang Z, Zhang B, He J, et al. AntiSARS-CoV-2 IgG levels in relation to disease severity of COVID-19. J Med Viro. 2022;94:380-3. DOI: 10.1002/ jmv. 27274
21 Chen JS, Alfajaro MM, Chow RD, Wei J, Filler RB, Eisenbarth SC, et al. Non-steroidal anti-inflammatory drugs dampen the cytokine and antibody response to SARS-CoV-2 infection. J Virol. 2021;95:e00014-00021. DOI: 10.1128/ JVI.00014-21, PMID: 33441348

22 Wisnewski AV, Campillo Luna J, Redlich CA. Human IgG and IgA responses to COVID-19 mRNA vaccines. PLoS One. 2021;16:e0249499. DOI: 10.1371/journal.pone.0249499, PMID: 34133415 